\title{
Intent-based Networking for Connectivity and Cloud Services
}

\author{
Mehmet Toy \\ Business Products, Verizon, Basking Ridge, New Jersey, USA
}

\section{Email address:}

Mehmet.toy@verizon.com

\section{To cite this article:}

Mehmet Toy. Intent-based Networking for Connectivity and Cloud Services. Advances in Networks. Vol. 9, No. 1, 2021, pp. 19-22. doi: $10.11648 /$ j.net.20210901.12

Received: February 13, 2021; Accepted: March 1, 2021; Published: March 10, 2021

\begin{abstract}
Recent developments in networking and services technologies increased number of choices for users and complexity of networks and services. At the same time, there is substantial effort in the industry to enhance user experiences with services. The enhancement of user experiences is tightly coupled with mapping of user intents to services by shielding customers from the complexity and automating management processes involved in ordering and delivering services. The mapping is an iterative process. Its accuracy is expected to be improved as Service Providers gain experience with the process. Automating management processes in a management infrastructure with non-virtualized OSSs (Operating Support Systems) is lengthy and difficult. Virtualization and artificial intelligence/machine learning (AI/ML) techniques accelerate the automation greatly. The automation improves not only user experiences with services, but also the management of networks and applications by network administrators. This paper will describe user intents, a method for mapping user intents to connectivity and cloud services, and the design process, with examples. The concepts and methods described here are expected to be used by consumers and administrators of networks and applications forming services. The intents and their mappings can be expanded for each service by adding unique capabilities and attributes for connectivity and application options of a given service.
\end{abstract}

Keywords: Networks, Services, Intent-based, Cloud, Mapping

\section{Introduction}

Recent developments in networking and services technologies increased number of choices for users and complexity of networks and services. In parallel to these developments, there has been substantial effort in the industry to enhance user experience with services. The enhancement of user experiences is tightly coupled with mapping of user intents to services by shielding customers from the complexity and by automating management processes.

Communications services can be divided as Connectivity and Cloud Services [1-4]. Connectivity Services are mostly offered as L1, L2 and L3 network services [5-7] such as Optical Transport, Carrier Ethernet, and IP services while Cloud Services are offered as Connectivity Services along with Cloud Applications such as Platform as a Service (PaaS), Infrastructure as a Service (IaaS) and Software as Service (SaaS). Cloud Applications are provided by Public Cloud Operators over Internet. They are also provided over private networks by Telecom Operators.

Intent-based Networking concept includes networking as well as applications although the term refers to networking only. The objective is to simplify operations for customers and network administrators by automating provisioning and full lifecycle management of the services.

Intent-based Networking (IBN) has been interpreted as deploying and configuring the network resources according to operator intentions automatically [10] or providing QoS level [11]. Similarly, [12] interprets IBN as allocating network resources from one or more networks, based at least in part on the desired performance parameters. The IBN use of Artificial Intelligence and Machine Learning algorithms to satisfy bandwidth requirements [13] and automate the network configuration [14]; and autoscaling of $5 \mathrm{G}$ platforms $[15,16]$ are also addressed.

This paper describes user intents for wireless and wireline Connectivity and Cloud Services, mapping user intents into all capabilities of Connectivity and Cloud Services, and the design process. 


\section{Process for Intent-based Networking}

The process for Intent-based Networking can be summarized as:

a. Identifying user intents based-on key words of the user for describing the desired service (s);

b. Mapping of the user intents to available services offered by the Service Provider based on an initial mapping template and using default values when there is no user input;

c. Presenting the solution resulted from the intent mapping to the customer and receiving additional customer inputs if needed;

d. Repeating steps (b) and (c) till finding the solution satisfactory to the user;

e. Configuring the network and application resources to implement the solution in (d); and

f. Updating the intent mapping template in (b) if needed.

These steps and a design process flow diagram are described in sections below.

\section{Intent Key Words}

Guessing key words that a user might use for requesting a service from a Service Provider is a difficult task. We group the key words for communication services as:

a. Service Type: Connectivity (e.g., wireless, wired, LAN, WAN, LAN and WAN), Application (e.g., Voice, Video, Messaging, File Transfer, Voice Conference, Video Conference, Holography, Security, etc.), Application and Connectivity, Wholesale, Retail;

b. Locations and Users: Number of Locations, Number of Users, Location Addresses, Location Type;

c. Security Level: High (e.g., Encryption, Block Chain) or Low;

d. Availability: High (e.g., five of 9s) or Low (e.g., Best Effort);

e. Service Level Agreement (SLA): Best Effort or performance constraints such as delay, jitter and loss that are critical for some applications such as self-driven cars and remote surgery services;

f. Elasticity: Service Duration, Immediate, Scheduled [8, 9]; and g. Billing Type: Fixed, Usage-based, and Per Transaction. These key words can be updated based on experience.

\section{User Inputs for Intent Identification}

From the keywords of Section 3, we can identify user intents as below:

a. Service Type $=$ \{connectivity $\Omega$ application $\Omega$ (connectivity and application) $\}$

b. Location-Connectivity $=\{$ location (s) requiring WAN connectivity $\Omega$ location (s) requiring LAN connectivity $\Omega$ (location (s) requiring LAN and WAN connectivity)

c. Location Type $=\{$ (number of locations $\leq 2) \Omega$ (number of locations $>2)\} \mathrm{U}$ \{(location type is commercial building) $\Omega$ (location type is campus) $\Omega$ (location type is stadium) $\Omega$ (location type is factory) $\Omega$ (location type is mobile)\}

d. Security $=\{$ high security $\Omega$ (medium to low security) $\}$

e. Availability $=\{$ high availability $\Omega$ (medium to low availability)\}

f. Service Level Agreement (SLA) $=\{$ (SLA with delay, jitter and loss constraints) $\cap$ Best Effort $\}$

g. Application Name

h. Application Tenancy $=\{$ Single or Multi Tenancy $\}$

i. Network Slicing $=\{$ Yes or No $\}$

j. Elasticity $=\{$ Yes or No $\}$

$\mathrm{k}$. Billing $=$ \{usage-based $\Omega$ fixed $\Omega$ per-transaction $\}$

From above, the complete user intent can be expressed as:

User Intent $=\{$ Service Type U Location-connectivity U Location type U Security U Availability U SLA U Application Name U Application Tenancy U Network Slicing U Elasticity U Billing\} EQ.1

where U represents "AND" function and $\Omega$ represents "OR" function.

\section{Service Model}

Services are modeled as Connectivity Services and Cloud Services as depicted in Figure 1. Connectivity Services are built over logical connections supported by network paths. The paths are formed of nodes such as switches and routers and physical links. Applications are built over Infrastructure supporting both network and applications.

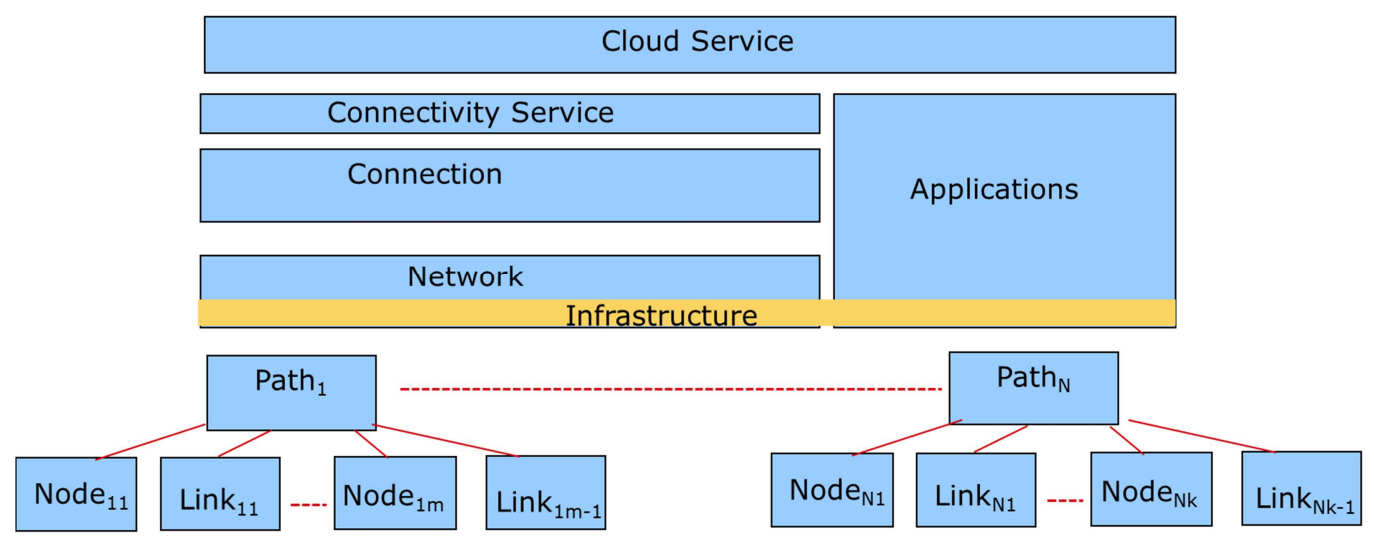

Figure 1. Service Modeling. 


\section{Connectivity Service Design Choices Based-on Intent}

Section 4 described how to determine the user intent. The next step is to map the user intent to Connectivity Services and Cloud Services consisting of connectivity and applications.

As an example, Table 1 maps some of the user intents in Section 4 into Connectivity Services. The user intents in Table 1 are:
a. High Security
b. High Availability
c. Best Effort
d. SLA
e. Service Type = connectivity
f. Location Type-Commercial Building

g. Location Connectivity- LAN Connectivity

h. Elasticity

i. Network Slicing

These intents are mapped into the following connectivity services in Table 1:

a. SD-LAN (software-defined LAN)

b. LAN-wired

c. LAN-wireless

d. LAN-WiFi

In order to map intents to Connectivity Services, for a given intent, each option is ranked as the first, second or third choice. For example, SD-LAN can be considered as the first choice for a High Security and SLA compliant LAN Connectivity Services. All services can support Elasticity and/or Network Slicing.

Table 1. An example mapping of user intents to Connectivity Services.

\begin{tabular}{llllll}
\hline $\begin{array}{l}\text { Connectivity } \\
\text { Service }\end{array}$ & User Intents & High Availability & Best Effort & SLA & $\begin{array}{l}\text { Location Type-Commercial } \\
\text { Building /w LAN Connectivity }\end{array}$ \\
\cline { 2 - 6 } & High Security & 1st choice with Redundant Path & N/A & N/A & 1st choice \\
\hline SD-LAN & 1st choice & 1st choice with Redundant Path & N/A & N/A & 2nd choice \\
LAN-wired & 2nd choice & 2nd choice & N/A & N/A & 2nd choice \\
LAN-wireless & 2nd choice & 2nd choice & N/A & N/A & 3rd choice \\
LAN-WiFi & 3rd choice & & &
\end{tabular}

\section{Connectivity Service Design Choices Based-on Intent}

A Cloud Service has both connectivity and application components [1-4]. Table 2 provides an example of mapping applications to Cloud Services. An application name along with SLA in addition to user intents for connectivity services are considered as the user intents for Cloud services in this example.

For example, an AR/VR/MR (Augmented Reality/Virtual Reality/Mixed Reality) Cloud Service requiring high security and availability with tight SLAs for applications could choose network slicing that does not use Internet for connectivity.

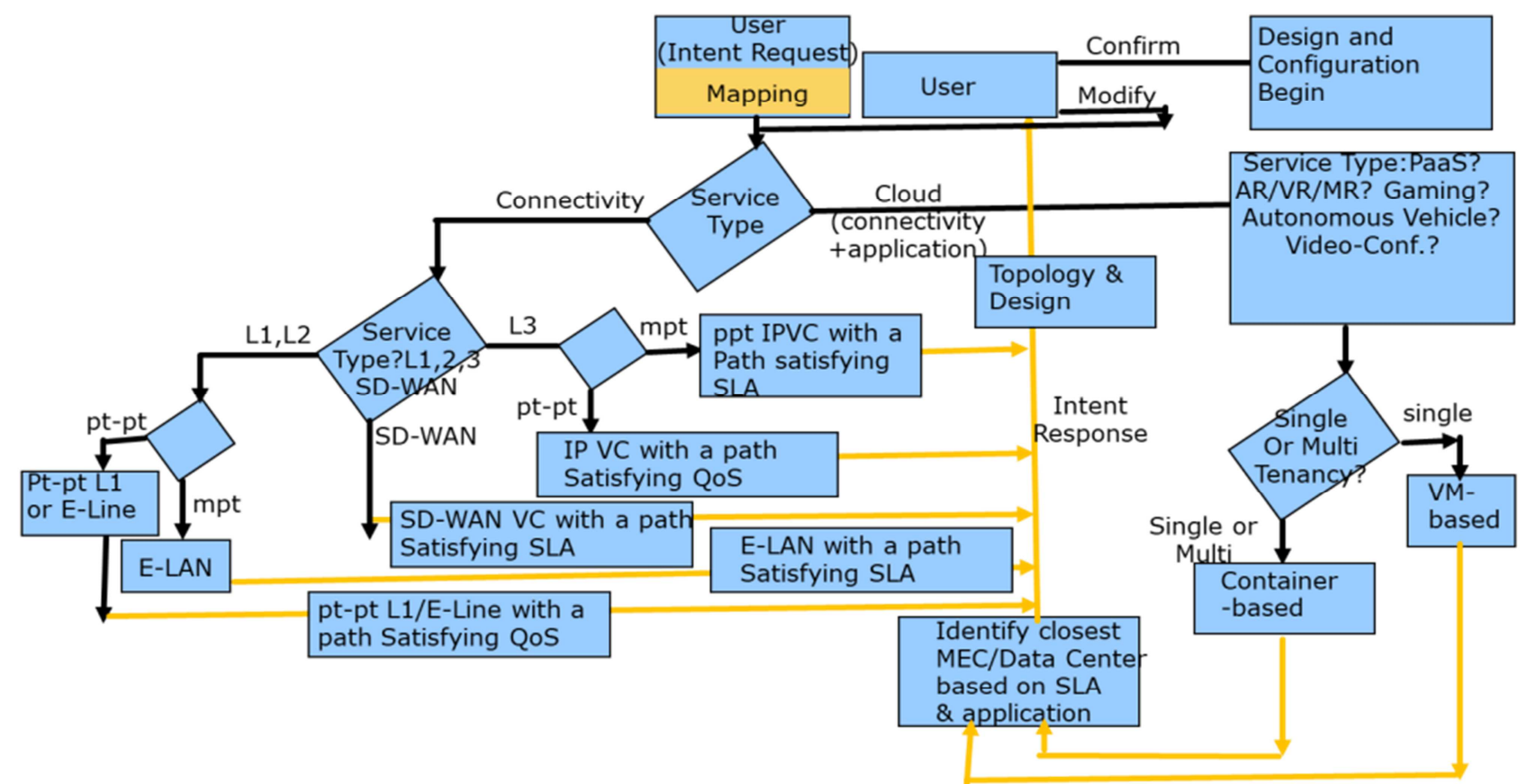

VM: Virtual Machine; pt-pt: Point-to-Point; mpt: Multi-point; VC: Virtual ConnectionCaaS: Communications as a Service

Figure 2. A Process for mapping user intents to services. 
Table 2. An example mapping of user intents to Cloud Services.

\begin{tabular}{|c|c|c|c|c|c|c|}
\hline \multirow{2}{*}{ Cloud Services } & \multicolumn{2}{|l|}{ AR/VR/MR } & \multicolumn{2}{|l|}{ Gaming } & \multicolumn{2}{|c|}{ Video Conferencing } \\
\hline & Best Effort & Support SLA & Best Effort & Support SLA & Best Effort & Support SLA \\
\hline Connectivity (non-internet)+ Application & 2nd choice & 2nd choice & 2nd choice & 2nd choice & 2nd choice & 2nd choice \\
\hline Network Slicing+ Application+ non-Internet for Connectivity & 3rd choice & 1st choice & 3rd choice & 1st choice & 3rd choice & 1st choice \\
\hline Connectivity (internet) + Application & 1st Choice & 3rd choice & 1st Choice & 3rd choice & 1st Choice & 3rd choice \\
\hline
\end{tabular}

AR/VR/MR-Augmented Reality/Virtual Reality/Mixed Reality

Science 61 (2015) 213-220.

\section{Service Design Process}

The process for mapping of user intents to services and designing services based on intents is depicted in Figure 2. The intents entered from a user portal or a user interface Application Programming Interface (API) are mapped to a service type (i.e., Connectivity Service or Cloud Service). With intents additional to those described in Section 4, a Connectivity Service or a Cloud Service is identified. The service is designed. The service topology is passed to the user for feedback. If the user intent is not met, the process is repeated.

The intended Connectivity Service, based on inputs in Section 4, is identified as LAN, L1, L2, L3 or SD-WAN. Depending on connection type and locations, it could be a point-to-point or a multipoint Connectivity Service. The next step is to determine Quality of Service (QoS) constraints from the intended SLA and identify connectivity paths accordingly.

If the intended service is a Cloud Service, its connectivity segment can be determined as above. Its application segment is determined based on Application Name, whether it is VNF (Virtual Network Function) or CNF (Cloud Native Network Function), whether it is for single tenant or multi-tenants, etc. In order to satisfy the QoS for applications, the applications maybe located at a Data Center or a Multi-access Edge Compute (MEC) location closest to the customer.

At the end, connectivity and application segments of the intended service is combined and presented to the customer for feedback. The process is repeated as needed.

\section{Conclusion}

In this paper, key words for user intents for Connectivity and Cloud services, forming user intents from these key words and mapping them to the services are described. The service design process based on intents is expected to be presented to the user for feedback before the implementation. A flow diagram for this process is given.

The intents and their mappings described here can be expanded to cover additional user intents, and capabilities of wireless and wireline networks and applications. They can be used as a base for the AI/ML techniques to be developed for IBN.

\section{References}

[1] OCC (Open Cloud Connect), "OCC 1.0 Reference Architecture", December 2014. https://wiki.mef.net/display/OCC

[2] M. Toy, "Cloud Services Architectures", Procedia Computer
[3] M. Toy, "MEFw68 Cloud Services Architecture", December 2020. https://wiki.mef.net/display/DSC/Cloud+Services+Architecture+ $\% 28 \mathrm{MEF}+\mathrm{w} 68 \% 29+-+$ Contributions

[4] ITU-T Technical Report 2020, "FGNET2030 Architecture Framework", July 2020.

[5] MEF, "MEF 63 Subscriber Layer 1 Service Attributes", August 2018.

https://www.mef.net/service-standards/underlay/optical-transport.

[6] MEF, "MEF 10.4 Subscriber Ethernet Services Attributes", December 2018. https://www.mef.net/service-standards/underlay/carrier-ethernet/

[7] MEF, "MEF 61.1 IP Service Attributes", May 2019. https://www.mef.net/service-standards/underlay/ip/

[8] M. Toy, "MEF76 Business Requirements and Use Cases for Access E-Line Service Control", April 2019. https://wiki.mef.net/display/LSO/Interlude+-+Access+E-Line + Service+Control+Contributions?preview $=\% 2 \mathrm{~F} 61965271 \% 2$ F88998011\%2FMEF+76+Letter+Ballot.doc

[9] M. Toy, "MEFw76 Business Requirements and Use Cases for Service Control", January 2020 L76040_005_MEF-W76-WD4.3-verizon-mtoy_Toy.pdf https://wiki.mef.net/pages/viewpage.action?pageId=121976548

[10] K. Abbas, M. Afaq, T. A. Khan, A. Rafiq and W. Song, "Slicing the Core Network and Radio Access Network Domains through Intent-Based Networking for 5G Networks" Electronics, 18 October 2020.

[11] M. Beshley; A. Pryslupskyi; O. Panchenko; H. Beshley "SDN/Cloud Solutions for Intent-Based Networking", 2019 3rd International Conference on Advanced Information and Communications Technologies (AICT).

[12] K. M. McBride, L. Tree, A. Jones, J. McManus, W. O ' Brien, Jr., C. M. Olson, "INTENT - BASED SERVICES.

[13] ORCHESTRATION", US Patent US 9, 882, 833 B2, Jan. 30, 2018.

[14] A. Mathew, "Artificial Intelligence for Intent Based Networking”, International Journal of Computer Science Trends and Technology (IJCST) - Volume 8 Issue 2, Mar-Apr 2020.

[15] T. A. Khan, A. Mehmood, J. J. D. Ravera; A. Muhammad; K. Abbas; W. Song, "Intent-Based Orchestration of Network Slices and Resource Assurance using Machine Learning", NOMS 2020 - 2020 IEEE/IFIP Network Operations and Management Symposium, 20-24 April 2020.

[16] J. Baranda, J. Mangues-Bafalluy; E. Zeydan, L. Vettori; R. Martínez, Xi Li, A. Garcia-Saavedra; C. F. Chiasserini, “ On the Integration of AI/ML-based scaling operations in the 5Growth platform", 2020 IEEE Conference on Network Function Virtualization and Software Defined Networks (NFV-SDN), 10-12 Nov. 2020. 